\title{
Inter Chip Fill for 3D Chip Stack
}

\author{
A. Horibe*, F. Yamada*, C. Feger** and J. U. Knickerbocker** \\ *IBM Research - Tokyo, 1623-14 Shimo-tsuruma, Yamato, Kanagawa 242-8502, Japan \\ **IBM Research - Thomas J Watson Research Center, 1101 Kitchawan Road, Yorktown Heights, NY 10598
}

(Received September 6, 2009; accepted November 24, 2009)

\begin{abstract}
Three-dimensional (3D) integration is considered to be the most promising solution for the continuing improvement in device performance,[1] while the scaling of $\mathrm{Si}$ CMOS is approaching its economical and physical limits. Inter Chip Fill (ICF) resin, filled between the gaps of 3D stacked chips, is expected to improve the mechanical strength and corrosion resistance of such chips. Pre-applied resin, which is applied before the chip joining process, was evaluated for this application. The characteristics required for ICF materials are different from those for conventional flip chip underfills, because the ICF must fill much thinner, multiple gaps of the order of a few micrometers between the silicon dies for which there is no CTE mismatch.

In this paper, a new ICF resin was designed for the 3D chip stack. The polymerization and viscosity of the material at each joining process step were precisely optimized. As a result, we could confirm the applicability of pre-applied ICF materials to the 3D chip integration process joined by very low height solder bumps of an area array. We arrived at the idea of a novel Stack Joining process which could be effective to reduce chip stacking process costs and contribute significantly to higher reliability.
\end{abstract}

Keywords: Inter Chip Fill, Stack Joining Process, Pre-Applied Resin, Fluxing Function, Adhesivity, 3D Chip Stack, Bump, Through Silicon Via

\section{Introduction}

Three-dimensional chip integration with through-siliconvias (TSV) and high-density metal joints is expected to provide higher intra-chip data bandwidth and lead to power-efficient, high-performance computing solutions such as servers and super computers. [2] 3D integration also gives higher bandwidth between heterogeneous chips such as imaging devices, DRAM, logic and analog chips. Higher performance $3 \mathrm{D}$ devices require finer pitch metal joints, which require thinner gaps between the stacked dies to keep the proper aspect ratio structures. When we are targeting $10 \mu \mathrm{m}$ pitch microjoints, the clearance between dies should be less than $10 \mu \mathrm{m}$. Figure 1 shows an example of 4 dies stacked in a 3D structure consisting of thin dies with TSV, metal bumps, filled resin between the dies and a plastic package. Such a 3D chip integrated device is required to have a robust, low-cost and high-yield process. In general, a chip stack is sequentially processed by putting a die on top of the substrate or previously stacked die, with alignment followed by high temperature soldering. As a result, the bottom die will experience the

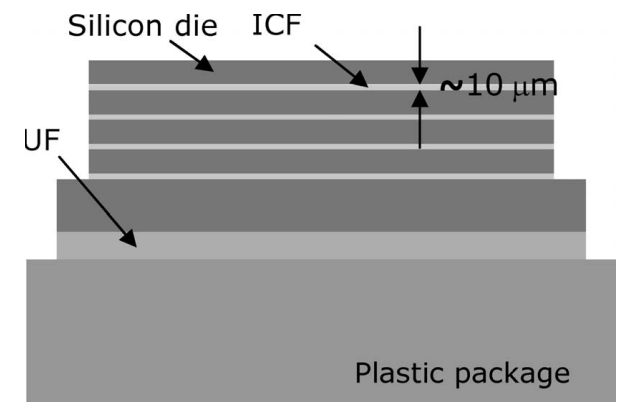

Fig. 1 Structure of 3D device.

number of heat cycles corresponding to the number of dies. The previously stacked solder joints will be melted repeatedly, unless the temperature hierarchy of the solders is considered. Inter Chip Fill resin is filled by the capillary fill process after all the chips are stacked.

With such a thin clearance, the capillary fill process takes a relatively long time, especially with larger dies, and the cleaning of flux residue becomes very difficult.

Pre-applied resin has already been developed as a wafer level underfill such as the "Over Bump Applied Resin (OBAR)” used for flip-chip packaging processes.[3] OBAR 
is intended for low-cost and high-reliability processes, by skipping both the capillary fill process to significantly reduce processing time, and the flux cleaning process by using a built-in fluxing capability.

In this paper, we studied the applicability of pre-applied ICF resin to a $3 \mathrm{D}$ integrated chip joined by very low profile solder bumps of an area array. We also extend the application of the ICF material to a novel Stack Joining process which could significantly reduce processing costs and improve reliability. This process uses a combination of repeated alignment with a low-temperature adhesion process, followed by a soldering process of all dies at a time.

This process could be applied not only to the chip-tochip process but also for the chip-to-wafer process.

\section{ICF and Stack Joining process}

\subsection{Inter Chip Fill}

The Inter Chip Fill we studied was a wafer level preapplied resin. A wafer coated with the ICF resin is diced into dies, and the dies are stacked using a flip-chip bonder. So, the pre-applied resin should not be sticky at room temperature. The glass transition temperature $(\mathrm{Tg})$ of the resin before post cure would be around $100^{\circ} \mathrm{C}$, and the resin is hardened (almost cured) at the bump joining temperature, which is about $230-260^{\circ} \mathrm{C}$ when lead-free solder is used as the joining metal.

\subsection{Stack Joining process concept}

Major concerns with multiple chip-stacking processes are the facts that they might involve long processing time and require repeated high-temperature treatments for joining the metal bumps between chips. The Stack Joining process which we propose can substantially reduce processing time and repeated high-temperature exposure by utilizing the adhesivity of the ICF at a temperature between the $\mathrm{Tg}$ and final curing temperature. In the Stack Joining process, multiple chips are temporarily stacked (without bump metal melt-joining) sequentially, and finally all the metal bumps of the stacked chips are melted and joined at once. The thinner resin layers help speed up this final bump melting process due to better heat conduction between the chips.

\subsection{Experiment}

Test vehicle dies with $10 \times 10 \times 0.07 \mathrm{~mm}$ and $150 \mu \mathrm{m}$ pitch area array bumps were used for the multiple diestacking experiment. The bumps are $80 \mu \mathrm{m}$ in diameter and $8.5 \mu \mathrm{m}$ high; and consist of $\mathrm{Cu}$ posts (5.5 $\mu \mathrm{m}$ high) with lead-free solder ( $3 \mu \mathrm{m}$ high) on the post. The pads consist of $\mathrm{Ni}(0.8 \mu \mathrm{m})$ and thin $\mathrm{Au}(0.1 \mu \mathrm{m})$. The passiva-
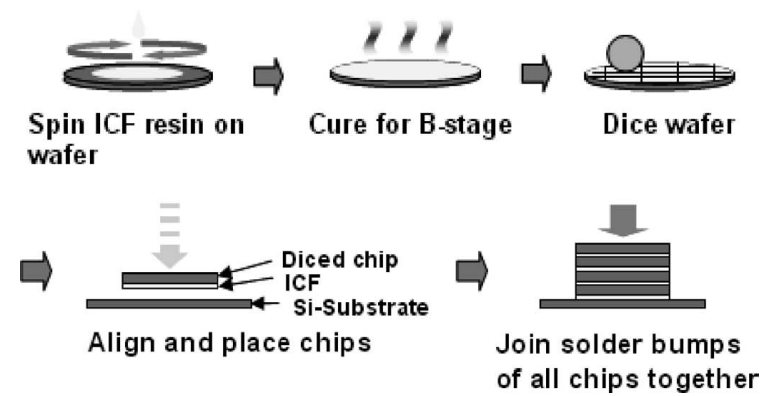

Fig. 2 Stack Joining process flow using B-stage-able ICF.

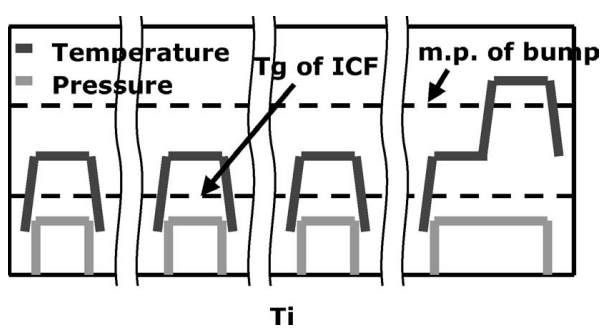

Fig. 3 Joining condition profiles for Stack Joining process.

tion openings under the bumps and pads are $40 \mu \mathrm{m}$ in diameter. Figure 2 shows a process flow of the Stack Joining process of a B-stage-able ICF and 4 die-stacking on a silicon substrate. The schematic joining condition profiles of temperature and pressure are shown in Figure 3. First, three dies were sequentially stacked and adhered at the stacking temperature which was lower than the melting point of the lead-free solder and higher than the Tg of the B-staged ICF. Then, the top-most die was placed on the stacked dies, heated above the melting point of the solder and pressed for metal bump joining of all dies. The ICF resin was fully cured during the final joining step.

\section{Results and Discussion}

\subsection{Stack Joining process results}

Figure 4 and Figure 5 show a cross-section of a bump joined by the Stack Joining process and a macro view of a

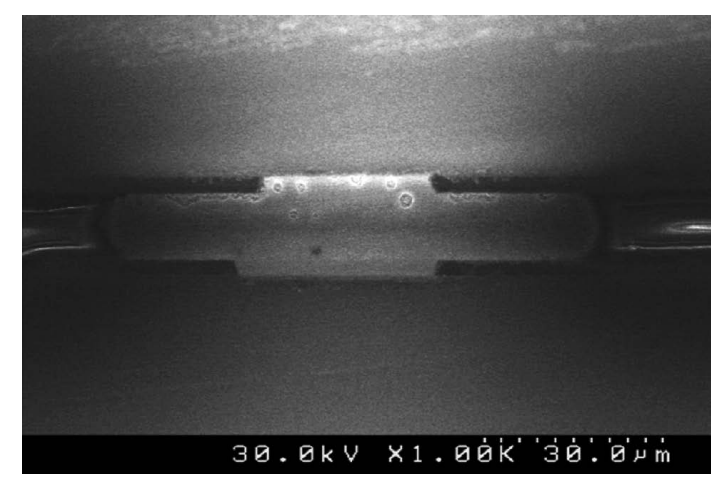

Fig. 4 Cross section of bump after Stack Joining. 


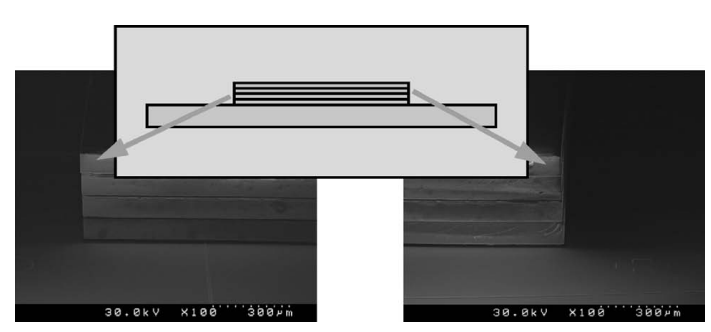

Fig. 5 3D chip after joining.

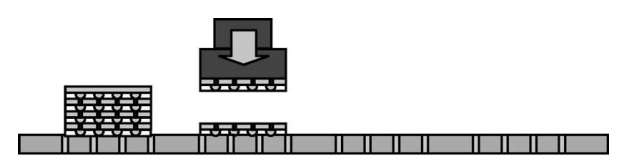

Stacking \& adhering dies on wafer

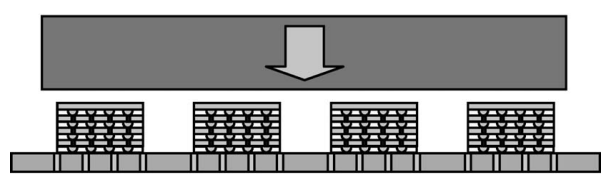

Joining all bumps

Fig. 6 Chip-to-wafer process by Stack Joining with ICF.

stacked chip observed using a scanning electron microscope (SEM). It can be seen that the adhesion of the Bstaged ICF resin keeps the alignment of the previously stacked dies true during the chip-stacking process. Electrical connection of all 3136 area array bumps of about $10 \mu \mathrm{m}$ high located between the bottom die and the silicon substrate was confirmed. (Electrical connections of upper chip was not measured because these dies have no TSVs.) The electrical resistance of each bump was about $30 \mathrm{mohm}$. This good joining result was achieved by precise optimization of the polymerization and viscosity control of the ICF resin at each process step. This Stack Joining process using the ICF can reduce processing time and hightemperature thermal cycle. This result, using $10 \mu \mathrm{m}$-high connections, also suggests that the Stack Joining process has potential for application to much finer pitch connections of less than $20 \mu \mathrm{m}$.

\subsection{Chip-to-wafer process applicability}

Figure 6 shows the chip-to-wafer process flow using ICF. Chip-to-wafer processing might have faster throughput than chip-to-chip. The Stack Joining process using ICF may be able to realize much faster, energy saving and reliable chip-to-wafer processing than a process without ICF due to the adhesive function at a lower temperature than melting-point of joining metal.

\section{Conclusions}

We confirmed that the ICF and the Stack Joining process are applicable to a 3D chip stack. The 3D chip stack process using this technique enables a significant reduction of processing time and improvement of $3 \mathrm{D}$ device reliability because the device does not experience the stress of thermal cycle associated with repeated soldering. The test vehicle with low-height bumps was fully joined electrically, which suggests that this technique has the potential for application to integration using finer pitch bumps such as 50 to $10 \mu \mathrm{m}$. Also, we propose a new chip-to-wafer process using Stack Joining with the ICF which would allow higher throughput and energy saving.

The 3D integrated device would have complex internal stresses caused by TSVs and the multi-layer structure. The proper ICF material development would be one of the potential approaches for the stress reduction and improvement of long-term reliability. As a next step, the long-term reliability of the 3D device filled with the ICF will be studied.

\section{References}

[1] www.itrs.net.

[2] J. U. Knickerbocker et al., "3D Silicon Integration”, $58^{\text {th }}$ Electronic Components and Technology Conference, pp. 538-543 (2008).

[3] C. Feger, N. C. LaBianca, H. K. Shobha, et al., “A wafer level underfill process for flip chip packaging”, Proc. IMAPS Topical Workshop and Exhibition on Flip Chip Packaging, Austin, TX, June (2003). 